\title{
A VERSION OF THE LEVY-BAXTER THEOREM FOR THE INCREMENTS OF BROWNIAN MOTION OF SEVERAL PARAMETERS ${ }^{1}$
}

\author{
SIMEON M. BERMAN
}

1. Introduction. Let $X\left(t_{1}, \cdots, t_{k}\right),-\infty<t_{1}, \cdots, t_{k}<\infty$, be Lévy's Brownian motion process of $k$ parameters: it is a Gaussian process with mean 0 and covariance function

$$
r(s, t)=\mathrm{E} X\left(s_{1}, \cdots, s_{k}\right) X\left(t_{1}, \cdots, t_{k}\right)=\frac{1}{2}[\|s\|+\|t\|-\|t-s\|]
$$

where $t=\left(t_{1}, \cdots, t_{k}\right)$ and $\|t\|=\left(t_{1}^{2}+\cdots+t_{k}^{2}\right)^{1 / 2}$. For each integer $n \geqq 1$, the unit cube $\left\{t: 0 \leqq t_{1} \leqq 1, \cdots, 0 \leqq t_{k} \leqq 1\right\}$ can be broken up in to $2^{n k}$ cubes whose edges have the common length $2^{-n}$. Such cubes have corner-points of the form $\left(i_{1} 2^{-n}, \cdots, i_{k} 2^{-n}\right)$, where the $i$ 's are in tegers between 0 and $2^{n}$. Put $i=\left(i_{1}, \cdots, i_{k}\right)$. Let us denote by $Y_{i, n}$ the $k$ th-order difference of the sample function $X$ over the cube $\left\{\boldsymbol{t}:\left(i_{1}-1\right) 2^{-n} \leqq t_{1} \leqq i_{1} 2^{-n}, \cdots,\left(i_{k}-1\right) 2^{-n} \leqq t_{k} \leqq i_{k} 2^{-n}\right\}=C(i, n)$ :

$$
\begin{gathered}
Y_{i, n}=\Delta_{1} \cdots \Delta_{k} X=X\left(i_{1} 2^{-n}, \cdots, i_{k} 2^{-n}\right)-\sum_{r=1}^{k} p_{r}+\sum_{r<s} p_{r s} \\
-\cdots+(-1)^{k} X\left(\left(i_{1}-1\right) 2^{-n}, \cdots,\left(i_{k}-1\right) 2^{-n}\right)
\end{gathered}
$$

where $p_{r s} \ldots t$ denotes $X\left(c_{1}, \cdots, c_{k}\right)$ for $c_{r}=\left(i_{r}-1\right) 2^{-n}$,

$$
c_{s}=\left(i_{s}-1\right) 2^{-n}, \cdots, c_{t}=\left(i_{t}-1\right) 2^{-n}
$$

and the remaining $c_{j}$ equal $i_{j} 2^{-n}$.

The result we shall prove is

Theorem. For $n \geqq 1$, let $\sum\left|Y_{i, n}\right|^{2 k}$ be the sum of the $2 k t h$ powers of the $Y_{i, n}$ over all cubes $C(i, n)$. Its limit, for $n \rightarrow \infty$, exists with probability 1 and is equal to a numerical constant $B_{k}$.

This represents a generalization of a classical theorem on the increments of the Brownian motion process of a one-dimensional time parameter, due to Lévy [2]. One can also extend this to a generalization of Baxter's theorem [1] to more general Gaussian processes of several parameters: indeed the proof of our theorem depends on the explicit form of the covariance only through the estimate in Lemma

Received by the editors October 4, 1966.

1 This paper represents results obtained at the Courant Institute of Mathematical Sciences, New York University, under the sponsorship of the National Science Foundation, Grant NSF-GP-6237. 
2.2 and the fact that the variance of $Y_{i, n}$ is bounded by a constant multiple of $2^{-n}$.

\section{Preliminary lemmas.}

Lemma 2.1. Let $X$ and $Y$ be random variables with a joint Gaussian distribution having means 0 , common variance $\sigma^{2}$, and correlation coefficient $\rho$; then

$$
\mathrm{E}\left\{X^{2 k} Y^{2 k}\right\}-\mathrm{E}\left\{X^{2 k}\right\} \mathrm{E}\left\{Y^{2 k}\right\} \leqq K \rho^{2} \sigma^{4 k}
$$

where $K$ is some numerical constant depending only on $k$.

Proof. It is sufficient to prove the inequality for the special case $\sigma=1$, as the general case follows from it. Suppose that a random variable $U$ has a Gaussian distribution with mean $m$ and variance $s^{2}$. An elementary computation shows that

$$
\mathrm{E} U^{2 k}=\sum_{j=0}^{k} c_{j} m^{2 j} s^{2(k-j)},
$$

for some constants $c_{0}, c_{1}, \cdots, c_{k}$ with $c_{0}=(2 k) ! / k ! 2^{k}$. The conditional distribution of $X$ given $Y$ is that of $U$ for $m=\rho Y, s^{2}=1-\rho^{2}$; therefore,

$$
\mathrm{E}\left[X^{2 k} \mid Y\right]=\sum_{j=0}^{k} c_{j} \rho^{2 j} Y^{2 j}\left(1-\rho^{2}\right)^{2(k-j)} .
$$

Multiply each side of this equation by $Y^{2 k}$ and take expectations:

$$
\begin{aligned}
\mathrm{E}\left[X^{2 k} Y^{2 k}\right] & =\sum_{j=0}^{k} c_{j} \rho^{2 j}\left(1-\rho^{2}\right)^{2(k-j)} \frac{[2(k+j)] !}{(k+j) ! 2^{k+j}} \\
& \left.=\left[(2 k) ! / k ! 2^{k}\right]\right]^{2}\left\{1-2 k \rho^{2}+P(\rho)\right\}
\end{aligned}
$$

where $P(x)$ is a polynomial whose terms of degrees 0 and 1 have coefficients 0 . The assertion of the lemma now follows from the inequality $|\rho|^{k} \leqq \rho^{2}$ for $|\rho| \leqq 1$ and $k \geqq 2$.

LeMMA 2.2. Put $r=\left(x_{1}^{2}+\cdots+x_{\mathbf{k}}^{2}\right)^{1 / 2}$; then

$$
\left|\frac{\partial^{2 k} r}{\partial x_{1}^{2} \cdots \partial x_{i}^{2}}\right| \leqq \frac{\text { constant }}{r^{2 k-1}}
$$

for all values of $x_{1}, \cdots, x_{k}$.

Proof. There are constants $c_{0 k}, \cdots, c_{k k}$ such that

$$
\frac{\partial^{2 k} r}{\partial x_{1}^{2} \cdots \partial x_{2 k}^{2}}=\sum_{j=0}^{k} c_{j k} r^{-[2(k+j)-1]} \sum_{1 \leqq i_{1}<\cdots<i_{j}: k} x_{i_{1}}^{2} \cdots x_{i_{i}}^{2},
$$


where the summation over $i_{1}, \cdots, i_{j}$ is understood to be 1 for $j=0$. This expression can be verified by induction on $k$; for this purpose, we note that derivatives of $r$ with respect to $x_{1}, \cdots, x_{j}$ depend on $x_{j+1}, \cdots, x_{k}$ only through $r$. It follows that the derivative on the left-hand side of the above equation is dominated by a constant multiple of

$$
r^{-(2 k-1)} \sum_{j=0}^{k} \sum_{1 \leqq i_{1}<\cdots<i_{j} \leq k} r^{-2 j} x_{i_{1}}^{2} \cdots x_{i_{i}}^{2} .
$$

The sum in the latter expression is bounded: for, on one hand, we have

$$
r^{-2 j} x_{i_{1}}^{2} \cdots x_{i_{j}}^{2} \leqq \frac{\left(x_{i_{1}} \cdots x_{i_{j}}\right)^{2}}{\left(x_{i_{1}}^{2}+\cdots+x_{i_{j}}^{2}\right)} j
$$

and, on the other hand,

$$
x_{i_{1}}^{2} \cdots x_{i_{j}}^{2} \leqq j^{-j}\left(x_{i_{1}}^{2}+\cdots+x_{i_{j}}^{2}\right)^{j}
$$

because the geometric mean never exceeds the arithmetic mean.

3. Proof of the Theorem. For $n \geqq 1$, the random variables $\left\{Y_{i, n}\right\}$ have a joint Gaussian distribution. The means are all 0 . Let us denote by $D_{k}$ the variance of the $k$ th-order difference of $X(\cdot)$ over the corner-points of the unit cube, i.e., the variance of $X(1, \cdots, 1)$ $-X(0,1, \cdots, 1)-\cdots-X(1, \cdots, 1,0)+X(0,0 ; 1, \cdots, 1)$ $+\cdots \pm X(0,0, \cdots, 0)$. The $Y_{i, n}$ have a common variance equal to $2^{-n} D_{k}$ : for, on one hand, the joint distribution of any finite collection of differences of the process $X$ is invariant under translations of the parameter set, by virtue of the form (1.1) of the covariance function, and so $Y_{i, n}$ has the same distribution as $Y_{0, n}$; and, on the other hand, $Y_{0, n}$ has the variance $2^{-n} D_{k}$ because the process $X\left(c t_{1}, \cdots, c t_{k}\right)$ is stochastically equivalent to the process $c^{1 / 2} X\left(t_{1}, \cdots, t_{k}\right)$, for any constant $c>0$. The covariance of $Y_{i, n}$ and $Y_{j, n}$ is equal to the $2 k$ th-order difference of the function $-\frac{1}{2}\|s-t\|$ over the product of the cubes $C(i, n)$ and $C(j, n)$. If the latter cubes are disjoint, then the difference is representable as the integral

$$
-\frac{1}{2} \int_{C(i, n)} \int_{C(j, n)} \frac{\partial^{2 k}\|s-t\|}{\partial s_{1} \cdots \partial s_{k} \partial t_{1} \cdots \partial t_{k}} d s_{1} \cdots d s_{k} d t_{1} \cdots d t_{k} .
$$

For proving the theorem it suffices to show that

$$
\mathrm{E}\left\{\sum_{i} Y_{i, n}^{2 k}\right\}=\frac{2 k !}{k ! 2^{k}} D_{k}^{k}=B_{k}
$$




$$
\sum_{n} \operatorname{Variance}\left(\sum_{i}\left|Y_{i, n}\right| 2 k\right)<\infty \text {. }
$$

The first relation is directly deducible from the distribution of $Y_{i, n}$; we shall shortly verify the second relation by showing that the variance of $\sum_{i}\left|Y_{i, n}\right|^{2 k}$ is dominated by a constant multiple of $2^{-n}$, $n=1,2, \cdots$.

In the rest of the paper we omit the subscript $n$ from $Y_{i, n}$, writing it as $Y_{i}$. The variance of $\sum_{i}\left|Y_{i}\right|^{2 k}$ is

$$
\sum_{i j}\left\{\mathrm{E}\left[Y_{i}^{2 k} Y_{j}^{2 k}\right]-\mathrm{E}\left[Y_{i}^{2 k}\right] \mathrm{E}\left[Y_{j}^{2 k}\right]\right\}
$$

There are $2^{2 n k}$ terms in this sum. Each term is dominated by a constant multiple of $2^{-2 n k}$; in fact,

$$
\mathrm{E}\left[Y_{i}^{2 k} Y_{j}^{2 k}\right] \leqq \mathrm{E}\left[Y_{i}^{4 k}\right]=2^{-2 n k} D_{k}^{2 k}[(4 k) ! /(2 k) !] 2^{-2 k} .
$$

There are at most $2^{2 n(k-1)}$ terms in the sum for which the indices are restricted by an equation of the form $i_{1}-j_{1}=\alpha_{1}$ for some integer $\alpha_{1}$. The total contribution of such terms to the sum cannot exceed a constant multiple of $2^{-n}$. The same is true of the total contribution of all terms whose indices satisfy at least one of the inequalities $\left|i_{1}-j_{1}\right|$ $\leqq 1, \cdots,\left|i_{k}-j_{k}\right| \leqq 1$. We complete the proof by showing that the contribution of all terms whose indices satisfy all of the inequalities

$$
\left|i_{1}-j_{1}\right|>1, \cdots,\left|i_{k}-j_{k}\right|>1
$$

does not exceed a constant multiple of $2^{-n k}$.

Lemma 2.1 implies that the general term of the sum (3.2) is bounded by a constant multiple of

$$
2^{-2 n k} \cdot\left[\operatorname{correlation}\left(Y_{i} Y_{j}\right)\right]^{2}=2^{-2 n(k-1)}\left[\operatorname{covariance}\left(Y_{i} Y_{j}\right)\right]^{2} \cdot \text { constant. }
$$

Lemma 2.2 and equation (3.1) imply that the covariance of $\left(Y_{i} Y_{j}\right)$ is dominated by a constant multiple of

$$
\begin{aligned}
& 2^{-2 n k} \max \left\{\|s-t\|^{-(2 k-1)}: s \in C(i, n), t \in C(j, n)\right\} \\
& =2^{-n}\left[\left(\left|i_{1}-j_{1}\right|-1\right)^{2}+\cdots+\left(\left|i_{k}-j_{k}\right|-1\right)^{2}\right]^{-(2 k-1) / 2} \text {; }
\end{aligned}
$$

thus, the general term of (3.2) whose indices satisfy (3.3) is dominated by a constant multiple of

$$
2^{-2 n k}\left[\left(\left|i_{1}-j_{1}\right|-1\right)^{2}+\cdots+\left(\left|i_{k}-j_{k}\right|-1\right)^{2}\right]^{-(2 k-1)} .
$$

The sum of all such terms is not greater than 


$$
\begin{aligned}
2^{-2 n k} \sum_{i_{1}, \cdots, i_{k}=1}^{2^{n}} & \left(2^{n}-1\right)^{k}\left[i_{1}^{2}+\cdots+i_{k}^{2}\right]^{-(2 k-1)} \\
& \leqq 2^{-n k} \sum_{i_{1}, \cdots, i_{k=1}}^{2^{n}}\left[i_{1}^{2}+\cdots+i_{k}^{2}\right]^{-(2 k-1)} .
\end{aligned}
$$

This is dominated by

$$
2^{-n k} k^{-(2 k-1)}\left(\sum_{\alpha=1}^{2^{n}} \alpha^{-4+(2 / k)}\right)^{k}
$$

because the geometric mean of $i_{1}^{2}, \cdots, i_{\mathbf{k}}^{2}$ does not exceed the arithmetic mean. The coefficient of $2^{-n k}$ in the above expression is bounded. The proof is complete.

I thank the referee for suggesting a few corrections to the first draft of this paper.

\section{REFERENCES}

1. G. Baxter, A strong limit theorem for Gaussian processes, Proc. Amer. Math. Soc. 7 (1956), 522-525.

2. P. Lévy, Le mouvement Brownien plan, Amer. J. Math. 62 (1940), 487-550.

Courant Institute of Mathematical Sciences, New York University 